\title{
A Method for the Identification of Mechanical Properties Using Surrogate Models
}

\author{
Leonardo Gutierrez, Han Li, Hiroyuki Toda, Masakazu Kobayashi, Osamu Kuwazuru, and Rafael Batres
}

\begin{abstract}
Identification of material properties involves physical experimentation followed by modeling, simulation and manual optimization. However, the last step tends to be computational expensive. This paper investigates an artificial neural network (ANN) surrogate model for identifying material parameters. The proposed approach is illustrated with a case study based on a nano-indentation test.
\end{abstract}

Index Terms-Surrogate models, optimization, metal-mechanic properties, infill sampling, inverse analysis.

\section{INTRODUCTION}

In material science and engineering, the estimation of material properties is associated to several applications including the detection of material failures, and the design of new materials. Identification of the "strongly-affecting materials characteristics" is certainly a key for improving the material design process [1].

Typically, the identification of material properties involves physical experimentation followed by modeling, simulation and manual optimization. In the manual optimization step, different sets of parameter values are proposed and simulations are performed. The optimization consists in determining which parameter combination matches best the physical experimentation data.

This paper focuses on the estimation of material parameters so that a further identification can be done quicker and cheaper than with conventional methods.

One conventional technique for estimating mechanical properties is to approximate a physical responseby means of a finite element analysis (FEA) model. Firstly, an experiment is carried out to obtain the physical response. Subsequently, the FEA model is executed several times in an attempt to reproduce the physical response. For each model execution, trial values are assigned to the model input variables (or parameters), then the model response is compared against the physical response (see Fig. 1). This method has been reported for the nanoindentation test [2], in which the problem consists on finding a FEA prediction that best fits the original experimental load-depth curve (see Fig. 2).

Although this methodology has proven to be effective in many cases, its success is highly influenced by the

Manuscript received November 5, 2013; revised January 14, 2014.

Gutierrez L., Li H. Kobayashi M., and Batres R. are with the Department of Mechanical Engineering, Toyohashi University of Technology, Toyohashi, Aichi 441-8580, Japan (e-mail: \{Leonardo, lihan, m-kobayashi\} @ ise.me.tut.ac.jp).

Toda H. is with the Department of Mechanical Engineering, Kyushu University, Fukuoka 819-0395, Japan (e-mail: toda@mech.kyushu-u.ac.jp).

Kuwazuru O. is with the Department of Nuclear Power \& Energy Safety, University of Fukui, Fukui 910-8507, Japan (e-mail: kuwa@u-fukui.ac.jp). availability of design experience. Furthermore, it is impractical when the number of parameters increases, since a larger number of simulations become necessary.

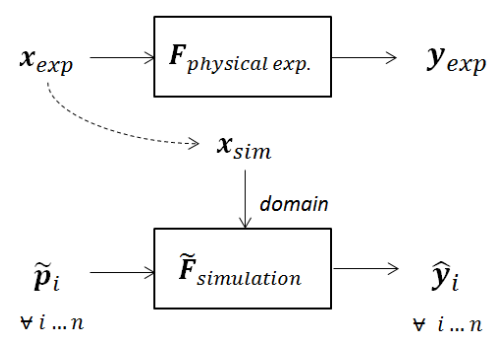

Fig. 1. Inputs and outputs for an experiment-simulation approach method. $x_{\text {exp }}$ and $x_{\text {sim }}$ are inputs, $y_{\text {exp }}$ and $\hat{y}_{i}$ are outputs and $\widetilde{\boldsymbol{p}}_{i}$ is a trial vector of parameters.

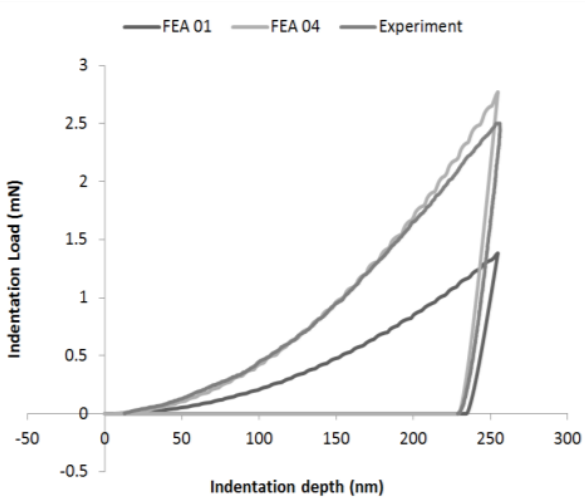

Fig. 2. Load-depth curve of a nano-indentation experiment and simulations of trial parameters (. FEA 01, FEA 04).

The proposed approach aims at carrying out as less simulation runs as possible which are used to generate a surrogate model that can be utilized "in lieu of the original computer model" to generate a response with less computation time. Thus, the surrogate model response can be compared against the experimental data and optimization techniques can be employed to find the desired values of the parameters. Surrogate models are constructed using data drawn from high-fidelity models, and provide fast approximations of the objectives and constraints at new design points, thereby making optimization studies feasible [3].Common surrogate modeling techniques include splines, polynomial approximation, Kriging models, radial basis functions, and artificial neural networks (ANNs).

Simpson et al. (2008) explain the growing usage of surrogate models [4]. Their review includes many developments in design and analysis of computer experiments (DACE) and new surrogate-modeling techniques proposed during recent decades. On the other hand, robust methodologies have been developed for calculating metal-mechanical properties [2]. However, very few attempts, such in [5], focused on computationally 
efficient methods. Furthermore, most of the existing literature concentrates in the physical interpretation of the results, and little has been done on approaches based on DACE.

Consequently, the objective of this research is to develop a systematic, general and robust methodology for determining material parameters within a minimum number of computer simulations, thus to lower the computational cost of conventional identification processes.

The rest of this paper is organized as follows. Section II describes the problem statement. The methodology is presented in Section III. Section IV illustrates the proposed approach with a case study for ananoindentation problem. Finally, the last section draws conclusions and areas of further research.

\section{Problem Statement}

The general problem can be stated as follows:

$$
\operatorname{Min}\left|\int_{x_{0}}^{x_{f i n a l}} \boldsymbol{F}(x, \boldsymbol{p}) d x-\int_{x_{0}}^{x_{f i n a l}} \widetilde{\boldsymbol{F}}(x, \widetilde{\boldsymbol{p}}) d x\right|
$$

subject to $g(x, \boldsymbol{p})=0$

where $\boldsymbol{F}(x, \boldsymbol{p})$ is the experiment response, and $\widetilde{\boldsymbol{F}}(x, \widetilde{\boldsymbol{p}})$ is a prediction obtained by a surrogate model.

$x$ is aknown independent variable, $\boldsymbol{p}$ is a vector of parameters which are intrinsically present in the material but whose range and values are unknown. In contrast, $\widetilde{\boldsymbol{p}}$ is a vector of parameters whose values are known a priori. $x_{0}$ is the initial value of $x$, and $x_{\text {final }}$ is its last value. The restriction $g(x, \boldsymbol{p})$ guarantees that the experimental and predicted responses overlap.

\section{Methodology}

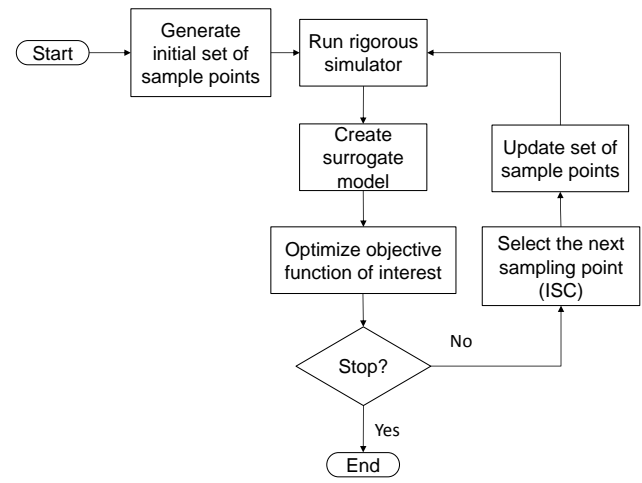

Fig. 3. Flowchart of a surrogate-based approach methodology with embedded ISC.

The methodology follows the super EGO algorithm proposed by Sasena et al. [6] which guarantees the creation of an accurate surrogate model. The algorithm works by first obtaining a set of sample points. Subsequently, rigorous simulations are performed for each sample point, and a surrogate model is fitted to those simulation results. The next step is to optimize the objective function of interest. If a termination criterion based on infill sampling criterion (ISC) is not satisfied, then the surrogate model is updated with the new sample points. Later the ISC is maximized to select the next sample points to be added. Lastly, the procedure is repeated until the termination criterion is reached. A flowchart of this methodology is shown in Fig. 3.

\section{A. Initial Data Sampling Strategy}

A finite number of sample points are needed to create a surrogate model. These points must be well-distributed in the design space.

In order to ensure the accuracy of the surrogate model in every region of the design space, various sampling techniques have been proposed. In this paper, we use the Latin hypercube sampling (LHS).

A variation of LHS is the optimum Latin hypercube (OLH) sampling. OLH employs the column pair wise (CP) algorithm [7] and generates an optimal design with respect to the $S$-optimality criterion. $S$-optimality seeks to maximize the mean distance from each design point to all the other points in the design, so the points are as spread out as possible along the design space.

\section{B. Creation of the Surrogate Model}

This step consists of performing simulations at the designed sampling points. Then the simulation responses are used for creating the surrogate model. A subsequent validation process based on a Bayesian metric can be used in order to decrease the natural uncertainty in surrogate models with limited number of design points [8].

In this paper we use ANNs as a surrogate method. Some of the advantages of ANNs are their versatility and simplicity. ANNs also provide better recognition of patterns in data and can result in better predictions of the response variables than conventional methods [9].

ANNs are computational models inspired by animal central nervous systems (in particular the brain) that are capable of machine learning and pattern recognition. They are usually presented as systems of interconnected "neurons" that can compute values from inputs by feeding information through the network. Three typical ANNs are back propagation, conjugate gradient, and Levemberg-Marquardt methods.

An ANN is trained by repeatedly presenting a series of input and output pattern sets to the network. The neural network gradually "learns" the relationship of interest by modifying the weights between its neurons to minimize the error between the actual and predicted output patterns of the training set. Then, a separate set of data called the test set is used to monitor network's performance. During training, the learning rule is used to iteratively adjust the weights and biases of the network in order to move the network outputs closer to the target values by minimizing the network performance indicator.

If many ANNs are trained, it becomes necessary to choose the best one by measuring their individual performance. The ability of surrogate models to reproduce the original model is commonly quantified by means of metrics such as the root-mean squared deviation (RMSD). The work in [8] and [9] shows that aBayesian metric can also be successfully applied to quantify the probability of data uncertainty in the prediction done by a set of surrogate models. Shi [8] employed the RMSD with $\ln Q$ Bayesian metric, and Noble 
[9] employed the Corrected Akaike's Information Criterion (AICc) and the Schwarz's Bayesian Criterion (SBC). A surrogate model is selected in terms of the smallest uncertainty (in the case of $\ln Q$-metric), or the lowest score (in the case of AICc and SBC).

\section{Optimization with Infill Sampling Criterion}

In order to improve the accuracy of the surrogate model and to facilitate the search of the global optimum, an approach using Infill Sampling Criterion (ISC) is proposed.

The purpose of ISC is to find the set of parameter values $\widetilde{\boldsymbol{p}}_{i}$, also called design variable, which maximizes the estimated error of the surrogate model predictor. Consequently, ISC searches for areas in the DS with high estimated error. To do this, ISC uses information of the current model in order to assess the utility of evaluating a design variable on the actual problem. The scope of infill criteria ranges from increasing the global accuracy of the surrogate model to facilitating the final optimization process.

An example of an ISC-based algorithm for Kriging surrogate models is the superEGO algorithm introduced by Sasena et al. [6]. Similar to superEGO, a proposed ISC approach is done for ANN.

The proposed ISC is based on the offset (error) value $I_{\tilde{F}}(\widetilde{\boldsymbol{p}})$, which is a quality index built upon the root mean squared deviation (RMSD) between the actual response and simulation data. It can be formulated as

$$
I_{\tilde{F}}(\widetilde{\boldsymbol{p}})=\sqrt{\frac{1}{m} \sum_{i=1}^{m}\left(\widetilde{\boldsymbol{F}}\left(x_{i}, \tilde{\boldsymbol{p}}\right)-\boldsymbol{F}\left(x_{i}, \boldsymbol{p}\right)\right)^{2}},
$$

where $\boldsymbol{F}\left(x_{i}, \boldsymbol{p}\right)$ is the $i^{\text {th }}$ actual experiment response at points $x_{i}$, and $\widetilde{\boldsymbol{F}}\left(x_{i}, \widetilde{\boldsymbol{p}}\right)$ is the surrogated prediction at this point, $m$ is the total number of experiment points.

We try to select a parameter design with the biggest contribution to the current error. In order to estimate the representativeness of a selected set $\widetilde{\boldsymbol{p}}_{\boldsymbol{i}}$, we introduce a weighted function

$$
w_{i, j}=d\left(\widetilde{\boldsymbol{p}}_{i}, \widetilde{\boldsymbol{p}}_{j}\right)
$$

where $\widetilde{\boldsymbol{p}}_{j}$ is the original OLH sampled data and

$$
d\left(\widetilde{\boldsymbol{p}}_{i}, \widetilde{\boldsymbol{p}}_{j}\right)=1-\exp \left(-\left\|\widetilde{\boldsymbol{p}}_{i}-\widetilde{\boldsymbol{p}}_{j}\right\|^{2}\right) .
$$

This weight function gives the similarity of the design variables. When the two design variables are near each other, the value of this function is small, and vice versa, the two design variables are far away, the value is big.

Since this criterion aims to maximize the difference of the experiment response and those infill variable designs that significantly deviate from the original OLH sampled data, the expression of the ISC becomes

$$
\widetilde{\boldsymbol{p}}_{i}=\operatorname{argmax}_{\widetilde{\boldsymbol{p}}_{i} \in D S} \sum_{j=1}^{n} w_{j}\left(\mathrm{I}\left(\widetilde{\boldsymbol{p}}_{i}\right)-\mathrm{I}\left(\widetilde{\boldsymbol{p}}_{j}\right)\right) .
$$

\section{CASE STUDY}

The experiment data is given by a nonlinear stress-strain relationship (hardening curve) in an aluminum-alloy material, which is obtained by means of a nanoindentation tester.
The nanoindentation experiment is carried out based on ISO14577-1 (2002) [10]. The experimental response is shown in Fig.4. The objective is to estimate the values of the elements of the vector of parameters

$$
\boldsymbol{p}=\{E, C, n, \alpha\}
$$

where $E$ is the Young's modulus, $C$ is the the strain-hardening coefficient, $n$ is the strain-hardening exponent and $\alpha$ is a strain constant. The determination of these parameters are often used to assess the mechanical reliability of materials (e.g. fatigue, fracture, corrosion and wear) [5], [11], [12].

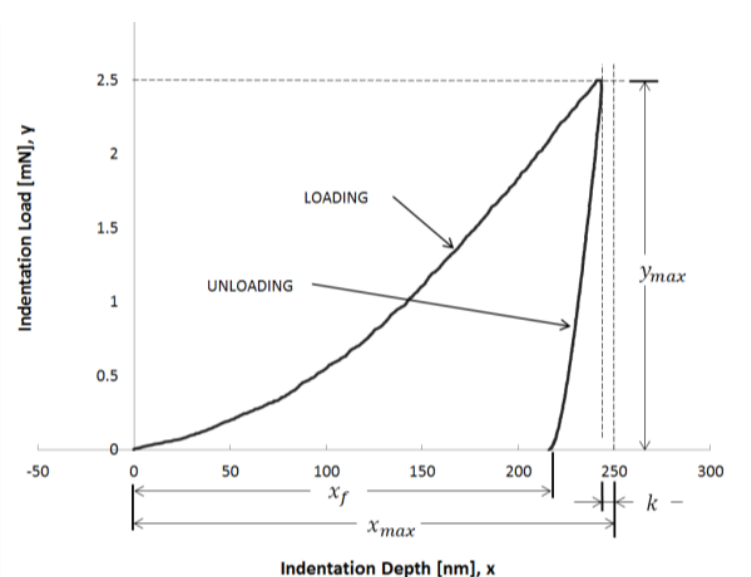

Fig. 4. Load-depth diagram for the case study indentation problem. $x_{f}$ : the final depth of the contact impression after unloading; $y_{\max }$ : the peak indentation load; $x_{\max }$ : the indenter ideal displacement at peak load; and $k$ : the post-experimental calibration.

The nanoindentation test is an extremely small-scale test carried out with nanometer order displacements, so the indenter tip is difficult to position exactly over the material surface at the beginning of the experiment. Hence, the indenter starts to move downward from above the material with some gap between the indenter tip and the surface. When the load cell senses the reaction force from the material surface, the displacement is set to zero and the force and displacement start to be recorded. However, the load signal includes some noise and the digital resolution of load is restricted, so it is difficult to sense exactly the touching force, and a delay may also occur. Moreover, the shape of the indenter tip is not guaranteed to be sharp enough, because the tip may become rounded by repeated experiments. The imperfection of the tip shape also affects the error and delay at the initial contact. Consequently, the indentation displacement is likely to be underestimated. As a result, the indentation displacement requires a calibration process that can correct the initial contact errors.

Here, a calibration factor $k$ is introduced as an unknown constant that represents the difference between an ideal maximum displacement $x_{\max }$ and the maximum measured displacement (see Fig. 4).

Since the peak of the indentation load-depth curve is noisy, the original response is regressed. Then the maximum real displacement becomes the convergence of a regression of both the load and the unload curves.

A classic procedure is to adopt a regression using the power law as suggested by Oliver and Pharr [5], [11]. The equation of the experimental response, including the calibration, is restated as 


$$
\boldsymbol{F}(x, \boldsymbol{p})= \begin{cases}A_{1}\left(x+C_{1}+k\right)^{n_{1}}, & x \in\left[x_{0}, x_{\max }\right] \\ A_{2}\left(x+C_{2}+k\right)^{n_{2}}, & x \in\left[x_{f}, x_{\max }\right]\end{cases}
$$

where $A_{i}, C_{i}$ and $n_{i}$ are the coefficients of the $i_{t h}$ curve regression, $x$ is the depth and $k$ is the calibration constant of the whole experimental response.

Since the maximum real displacement is considered to be the intersection of the loading with the unloading regressed curves, the value of $k$ can be calculated with the least squares method (LS) using a general purpose optimizer. This intermediate problem consists in solving

$$
k=x_{\max }-\min _{x}\left|A_{1}\left(x-C_{1}\right)^{n 1}-A_{2}\left(x-C_{2}\right)^{n 2}\right|,
$$

where the subscript 1 refers to the coefficients that belongs to the loading regression and 2 refers to the unloading regression.

\section{A. Sampling Strategy}

Because OHS is intended for box-like domains, a minimum and a maximum limits for each of the sought parameters must be specified. These upper and lower limits constitute fixed region in the design space. The parameter limits are shown in Table I.

TABLE I: DESIGN SPACE OF INPUT VARIABLES

\begin{tabular}{lllll}
\hline \hline Parameters & $E(\mathrm{GPa})$ & $C(\mathrm{MPa})$ & $n$ & $a$ \\
\hline Lower Limit & 50 & 500 & 0.1 & 0 \\
Upper Limit & 150 & 1500 & 0.3 & 0.002 \\
\hline \hline
\end{tabular}

In order to simplify the problem, in this case study we assume that the set of sample points satisfies the infill sampling criterion described in Section III.

TABLE II: LHS OF 8 PARTITIONS, 4 VARIABLES

\begin{tabular}{crrrr}
\hline \hline Parameters & $E(\mathrm{GPa})$ & $C(\mathrm{MPa})$ & \multicolumn{2}{l}{$a$} \\
\hline 1 & 55.31471 & 611.9684 & 0.218075 & $5.24 \mathrm{E}-04$ \\
2 & 132.059 & 549.7711 & 0.163307 & $7.85 \mathrm{E}-04$ \\
3 & 75.79828 & 715.9358 & 0.143577 & $1.94 \mathrm{E}-03$ \\
4 & 89.83298 & 848.659 & 0.108379 & $4.97 \mathrm{E}-04$ \\
5 & 119.3931 & 656.3377 & 0.265272 & $1.52 \mathrm{E}-03$ \\
6 & 144.2803 & 923.2771 & 0.198678 & $1.21 \mathrm{E}-03$ \\
7 & 108.1492 & 769.9889 & 0.291477 & $1.00 \mathrm{E}-07$ \\
8 & 69.99183 & 947.56 & 0.225471 & $1.35 \mathrm{E}-03$ \\
\hline \hline
\end{tabular}

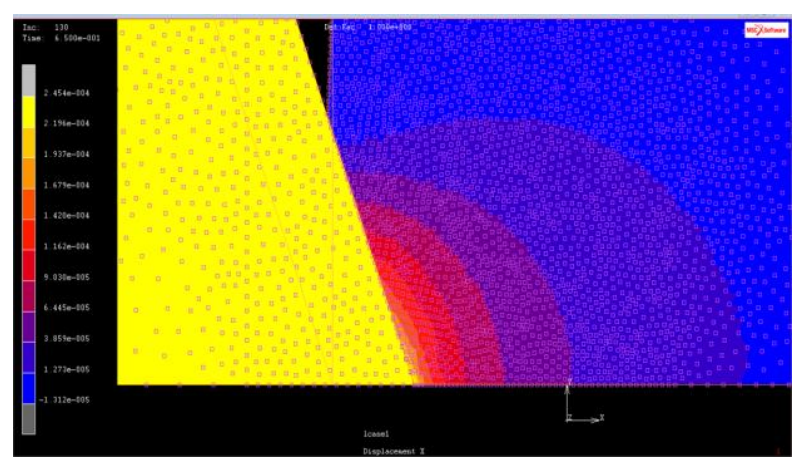

Fig. 5. Indenter (yellow), and Aluminum material (blue) in the FEM in 2D through interface in MARC MENTAT®. Colors represent the scalar deformation in the contour of the mesh.

The design of experiments was performed using optimum LHS included in the 'LHS' package of ' $\mathrm{R}$ ' statistical programming environment. The generated set of sampling points is shown in Table II.

Subsequently, simulations were carried out on those points using MARC@ simulation software [13]. The numerical data from the FEA is used for training and verification of the ANN (see Fig. 5).

\section{B. Creation of the Surrogate Model}

In this research, six parameters are considered as input variables. Four of them represent the material properties, one is the independent variable (displacement) of the experiment and one more variable $b$ is a binary artifice that helps to separate the prediction into two sections(load and unload).For computational purposes, the surrogate model restated function is

$$
\widetilde{\boldsymbol{F}}(x, \widetilde{\boldsymbol{p}}) \equiv \widetilde{\boldsymbol{F}}(x, b, \widetilde{\boldsymbol{p}}) .
$$

In (9), $b=0$ represents the loading section of the curve and $b=1$ is for the unload section of the curve of Fig. 3 .

ANNs were created using the Neuroet toolbox [9]. The optimum number of neurons in the hidden layer is obtained via a built-in function in Neuroet.

Additionally, in order to select an adequate surrogate model, a statistical analysis based on the SBC score was carried out. The criterion consisted in selecting the ANN features which have the highest probability of being included in the top $5 \%$ among $3 \times 15 \times 18 \times 12$ ANNs' SBC scores ${ }^{1}$.

The analysis showed that for the nano-indentation response (the load-depth curve), an ANN trained with 8 hidden layers using the standard back-propagation method produced the smallest SBC. Furthermore, the transfer function between the input layer and the hidden layer was set to log-sigmoid, while the transfer function between the hidden layer and the output layer was pure-linear.

\section{Restatement of the Objective Function}

In the objective function, it is important to consider that the experiment is noisy and also could miss certain information or cluster information in determined sections of the curve. The first term in (1) that refers to the integration of the experimental curve is approximated through the integration of (7) as follows

$$
\begin{gathered}
\int_{u_{0}}^{u_{\text {final }}} \boldsymbol{F}(x, b, \boldsymbol{p}) d x \approx \int_{x 0}^{x \max } A_{1}\left(x+C_{1}\right)^{n 1} d x \\
+\int_{x \max }^{x f} A_{2}\left(x+C_{2}\right)^{n 2} d x
\end{gathered}
$$

where the coefficients $A_{1}, C_{1}$ and $n_{1}$ belong to a regression of the load curve and coefficients $A_{2}, C_{2}$ and $n_{2}$ belong to the unload curve. $x_{\max }$ is the maximum depth (Fig. 1).

The second term of (1) is approximated as

$$
\int_{x_{0}}^{x_{\text {final }}} \widetilde{\boldsymbol{F}}(x, \widetilde{\boldsymbol{p}}) d x \approx \sum_{i=0}^{n} \frac{\left(x_{i}-x_{i-1}\right) *\left(\hat{y}_{i}+\hat{y}_{1-1}\right)}{2}
$$

1 In the analysis we compared 3 training methods (Standard Backpropagation, Conjugate Gradient, and LevembergMarqardt), a best selection among 15 trainings for each of the 18 candidates for number of hidden layers ( 1 to 18 ), and a final of 12 runs of the whole process selection. 
where $\widehat{y}_{i}$ is the surrogate model prediction in $x_{i}$.

Finally, the restriction $g(x)$ is implemented as a composed penalty function:

$$
g(u)=\omega_{1} *\left|A_{1}\left(x_{\max }+C_{1}\right)^{n 1}-\widetilde{\boldsymbol{F}}\left(x_{\max }, 0, \widetilde{\boldsymbol{p}}\right)\right|
$$

where $\omega_{1}$ is a weight which can be adjusted manually, the term $A_{1}\left(x_{\text {max }}+C_{1}\right)^{n 1}$ is the peak of the regressed loading curve, and the term $\widetilde{\boldsymbol{F}}\left(x_{\max }, 0, \widetilde{\boldsymbol{p}}\right)$ stands for the peak of the surrogate model loading curve.

The constraint represents a weighted difference between both the experimental curve and predicted curve peaks. The value of $\omega_{1}$ is likely to vary depending on the scale of the prediction, or number of parameters (other features, such as the dispersion of the original response, could influence it too). The weight $\omega_{1}$ gave satisfactory results for this case study (4 parameters with a maximum depth of $x_{\max }=$ $250[\mathrm{~nm}]$ ) for the range $0.1 \leq \omega_{2} \leq 0.3$.

\section{Optimization}

In the proposed methodology, the purpose is to strategically find a combination of inputs which solves (1). Several stochastic optimization algorithms can be used. Since (1) is a non-linear function, a global optimizer can address this problem. We used differential evolution algorithm (DE), [14] to find the optimum material parameters.

\section{E. Results}

The calculations were carried out with $\mathrm{R}$, on a Core 2 Duo computer, running Windows 7 32bits. The optimization was run 10 times and it took almost 4 minto complete each one. The results are shown in Table III. Additionally, in order to validate these results, we compared them to a manually adjusted FEA procedure based on [2].

TABLE III: RESULTS OBTAINED WITH ANN VS A MANUAL FITTING

\begin{tabular}{lrrrr}
\hline \hline Parameters & $E(\mathrm{GPa})$ & $C(\mathrm{MPa})$ & $n$ & $\alpha$ \\
\hline $\begin{array}{l}\text { Optimized } \\
\text { (mean) values }\end{array}$ & 82.95 & 820.9 & 0.1308 & $2.680 \mathrm{E}-4$ \\
$\begin{array}{l}\text { Standard deviation } \\
\text { Manually fitted } \\
\text { values }\end{array}$ & 0.07 & 3.0 & 0.001 & $0.296 \mathrm{E}-4$ \\
\hline \hline
\end{tabular}

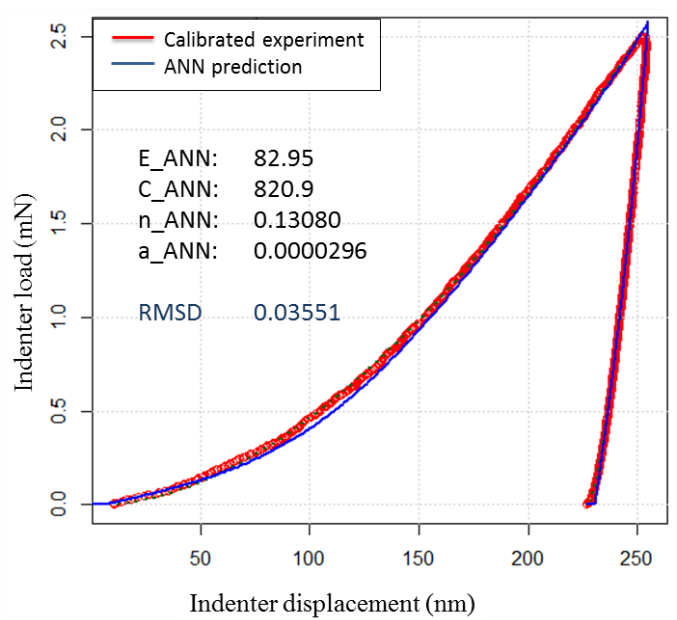

Fig. 6. Load-depth curve of the physical experiment versus a prediction generated through ANN method.

The conventional manual fitting required from material-science experts to perform between 10 to 20 simulations, each one taking approximately 30 minutes in MARC® on a HP Intel Xeon workstation.

Fig. 6 shows two curves: a) the physical experiment and b) the optimized surrogated prediction. In the ANN, the optimum had an RMSD $=0.03551(\mathrm{mN})$.

Finally, we made two FEM simulations using both the optimized mean values, and the manually fitted values from Table III. Fig. 7 shows the FEM simulation using the proposed methodology prediction. Table IV contains the actual RMSD of both curves, each one versus the physical experiment response.

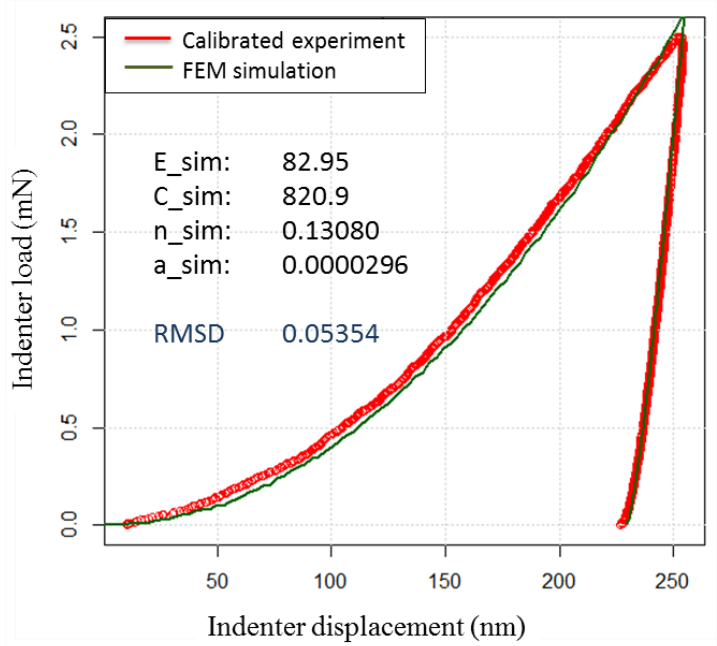

Fig. 7. Load-depth curve of the physical experiment versus a simulation using the surrogate-based optimum parameters.

TABLE IV: ACCURACY OF ANN VS MANUAL FITTING

\begin{tabular}{lc}
\hline \hline Simulated curves & $R M S D(\mathrm{mN})$ \\
\hline Optimized values & 0.0535 \\
Manually fitted values & 0.1349 \\
\hline \hline
\end{tabular}

\section{CONCLUSION}

A method was proposed for the identification of mechanical properties of materials. The results point out that surrogate models can be used together with an optimization algorithm to identify material parameters. A case study was presented for the estimation of material parameters obtained from a nanoindentation load-depth curve and a small number of computer simulations. The proposed systematic methodology improves the computational and time costs, and also the accuracy of the existing methods.

Further work includes exploring other surrogate-modeling techniques such as Kriging or Support Vector Machine, and comparing. Also a changeable design space is worth considering. Finally, we plan to investigate more complex problems involving a larger number of material parameters.

\section{ACKNOWLEDGMENT}

We thank Professors Yoshiaki Shimizu, Tatsuhiko Sakaguchi and Vinicius Aguiar for their valuable advice.

The work carried out in this paper was supported by a grant from the Japan Society for the Promotion of Science under Grant-in-Aid for Scientific Research S No. 24226015. This work was financially supported by JSPS KAKENHI Grant Number 24226015. 


\section{REFERENCES}

[1] H. Toda, S. Masuda, R. Batres, M. Kobayashi et al., "Statistical assessment of fatigue crack initiation from sub-surface hydrogen micropores in high-quality die-cast aluminum," Acta Materialia, vol. 59, pp. 4990-4998, 2011.

[2] J. L. Bucaille, S. Stauss, E. Felder, and J. Michler, "Determination of plastic properties of metals by instrumented indentation using different sharp indenters," Acta Materialia, vol. 51, pp. 1663-1678, 2003.

[3] N. V. Queipo, R. T. Haftka, W. Shyy, T. Goel, R. Vaidyanathan, and P. T. Kevin, "Surrogate-Based analysis and optimization," Progress in Aerospace Sciences 41, pp. 1-28, 2005.

[4] T. W. Simpson, V. Toropov, V. Balabanov, and F. A. C. Viana, "Design and analysis of computer experiments in multidisciplinary design optimization: A review of how far we have come - or not," American Institute of Aeronautics and Astronautics, 2008.

[5] H. Jin, W. Yang, and L. Yan, "Determination of residual stresses and material properties by an energy-based method using artificial neural networks," Estonian Academy of Sciences, pp. 296-305, 2012.

[6] M. Sasena, P. Papalambros, and Goovaerts, "Global optimization of problems with disconnected feasible regions via surrogate modeling," American Institute of Aeronautics and Astronautics, 2002.

[7] J. S. Park, "Optimal latin-hypercube designs for computer experiments", Journal of Statistical Planning and Inference, vol. 39 pp. 95-111, 1994.

[8] L. Shi, R. J. Yang, and P. Zhu, "A method for selecting surrogate models in crashworthiness optimization," Struct Multidisc Optim, vol. 46, pp. 159-170, 2012.

[9] A. P. Noble and H. E. Tribou, "Neuroet: An easy-to-use artificial neural network for ecological and biological modeling," Ecological Modeling, vol. 203, pp. 87-98, 2007.

[10] Metallic Materials - Instrumented Indentation Test for Hardness and Materials Parameters - Part 1: Test method, ISO14577-1, 2002.

[11] W. C. Oliver and G. M. Pharr, "An improved technique for determining hardness and elastic modulus using load and displacement sensing indentation experiments," Journal of Material Research, vol. 7, pp. 1564-1583, 1992.

[12] X. Chen, J. Yan, and A. M. Karlsson, "On the determination of residual stress and mechanical properties by indentation," Materials Science and Engineering A 416, pp. 139-149, 2006.

[13] MSC.MARC Software, MARC user's guide. (2008). [Online]. Available: http://simcompanion.mscsoftware.com/infocenter/index

[14] D. Ardia, K. Boudt, P. Carl, K. Mullen, and B. G. Peterson, "Differential evolution with DEoptim, an application to non-convex portfolio optimization," The R journal, vol. 3, no. 1, pp. 27-34, 2011.

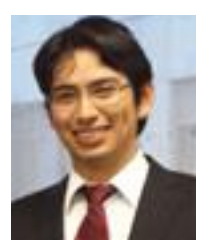

Leonardo Gutierrez received his BS in industrial engineering from the National Polytechnic Institute in 2012. Currently, Leonardo is pursuing his master's degree in mechanical engineering at Toyohashi University of Technology in Japan. He worked as Jr. Functional Oracle E-Business Suite Consultant for SIR Tecnologia S.A., outsourced in Grupo Aeromexico S.A., Oracle Mexico S.A., Grupo GEO S.A. and The Solocup Company Mexico S.A. until 2012. His current research interests include computer-aided decision making and optimization. Mr. Gutierrez belongs to the Industrial Systems Engineering Group in Toyohashi University of Technology.

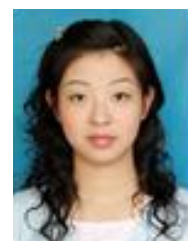

Han Li received BS degree in mathematics and applied mathematics from Faculty of Mathematics and Computer Science in Hubei University in 2007. She received her Ph.D. degree in the School of Mathematics and Statistics at Beijing University of Aeronautics and Astronautics. Her research interests include neural networks, learning theory and pattern recognition. She now works as a researcher in the Department of Mechanical Engineering, Toyohashi University of Technology. Dr. Li belongs to the Industrial Systems Engineering Group in Toyohashi University of Technology.

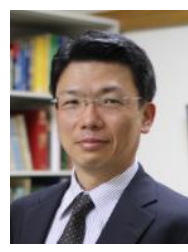

Hiroyuki Toda graduated from Kyoto University in 1987, and received his doctor of engineering degree in 1995. He is currently a distinguished professor in structural materials and a director of the 3D/4D Structural Materials Research Centre in Kyushu University. His research interests are 3D/4D image-based analysis and its application to understand microstructure-properties relationships in engineering materials.

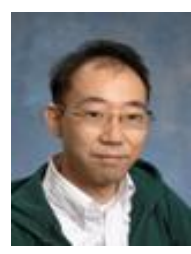

Masakazu Kobayashi received his bachelor of engineering degree from the Faculty of Engineering, Utsunomiya University in 1996, his master of engineering degree in 1998 and his doctor of engineering degree in 2001 also from Utsunomiya University, Japan. His major is development of $3 \mathrm{D}$ analysis method for material microstructure and its application. He worked as a researcher at Utsunomiya University before moving on to Toyohashi. He currently works as an associate professor at the Department of Mechanical Engineering, Toyohashi University of Technology

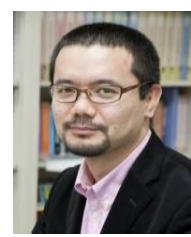

Osamu Kuwazuru received his Bachelor of Engineering degree from the Faculty of Engineering, Shibaura Institute of Technology in 1996, his Master of Engineering degree in 1998 and his Doctor of Engineering degree in 2001 from the University of Tokyo. His research interests include computational solid mechanics, nonlinear numerical modeling, 4D imaging, fatigue, corrosion, and micromechanics. He worked as an assistant professor at Institute of Industrial Science, the University of Tokyo between 2001 and 2008, and currently works as an associate professor at University of Fukui since 2008. Dr. Kuwazuru belongs to the Department of Nuclear Power \& Energy Safety Engineering, University of Fukui.

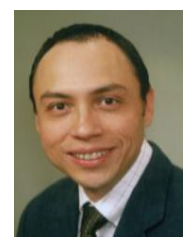

Rafael Batres is an associate professor of industrial and systems engineering in the Mechanical Engineering Department at Toyohashi University of Technology. Hereceived a BS in chemical engineering from the National University of Mexico (UNAM) and both a MEng and PhDin process systems engineering from Tokyo Institute ofTechnology. His research is currently focused on material and product design, design and simulation of sustainable systems, systematic methods for ontology development, and large-scale data management and analytics. 